\title{
Écologie d'une rivière non aménagée des Alpes du Sud : Le Buëch (France) \\ II. Action d'un flux polluant sur l'hydrochimie du cours d'eau
}

F. Vespini 1

P. Légier ${ }^{2}$

A. Champeau 1

Mots clés : hydrochimie, effluents, rivière, Alpes, analyse en composantes principales.

Dans son secteur moyen, le Buèch draîne les eaux de rebut de la ville de Laragne. L'évolution spatiale du chimisme des eaux usées est étudiée au niveau de 6 stations du réseau d'égouttage et du Véragne (cours d'eau collecteur des effluents de la ville et de son abattoir). Son évolution temporelle est suivie toutes les heures pendant une journée. L'impact des rejets sur le Buëch est analysé à partir de prélèvements réalisés dans cinq stations prises en amont et en aval de la confluence du Buëch et du Véragne.

Sévère à l'échelle de l'affluent, la pollution est peu apparente à l'échelle du cours principal. L'étude met en évidence l'importance prépondérante des phénomènes de dilution et de sédimentation sur le phénomène de biodégradation, ce dernier n'étant vraiment décelable que pendant la période de l'étiage estival.

Ecology of a freeflowing river of the southern Alps : the Buch (France) II - Action of a polluting flux on the water's hydrochemistry

Keywords : hydrochemistry, effluents, river, Alps, principal component analysis.

In its middle section, the Buëch river drains waste water from the city of Laragne. The spatial development of the wastewater's chemical nature was studied at 6 points on the sewer drainage system and the Veragne river (river receiving waste water outflow from the city and its slaughterhouse). Temporal evolution was noted for each hour of the day. The impact of waste on the Buëch river was analyzed through samples taken at 5 points upstream and downstream from the confluence of the Buëch and Veragne rivers.

Though high in its tributary, pollution is rather low in the river itself. The study makes clear the over-whelming importance of dilution and sedimentation on biodegradation, the latter being barely detectable during the summer dry season.

\section{Introduction}

Un suivi écologique mené sur le Buëch, rivière des Alpes du Sud, a mont ré que les composantes physi. ques et chimiques du cours subissent une évolution " naturelle " de l'amont vers l'aval. A cette eutrophi. sation se superpose une eutrophication résultant du

\footnotetext{
Travail réalisé avec la collaboration technique de MM. Habai (J.C.) et Garnier (R.).

1. Laboratoire d'Hydrobiologie, Université d'Aix-Marseille I, Centre Saint-Charles, Marseille.

2. Laboratoire d'Ecologie Animale, Hydrobiologie, Université d'Aix-Marseille III, centre Saint-Jérôme, Marseille.
}

rejet des eaux usées des deux principales villes riveraines : Serres et Laragne. (Vespini, Légier \& Champeau 1987). Les perturbations apportées par la ville de Laragne et par ses abattoirs sont importantes en intensité ponctuelle. Leurs répercussions qui se font sentir sur le cours inférieur du Buëch font l'objet d'une étude détaillée dans cette deuxième note' ${ }^{\text {. }}$. Avant d'aborder l'étude du cours axial du Buëch, il convient de définir le rejet lui-même et pour cela d'analyser :

\footnotetext{
1. La numérotation des stations correspond à celle utilisée par les auteurs dans la publication citée précédemment.
} 
- sa composition au sortir du réseau d'égout tage et son évolution spatiale dans le Véragne en amont de la confluence avec le Buëch ;

- son évolution journalière au travers des principaux paramètres liés aux activités domestiques.

\section{Méthodes}

\subsection{Composition et évolution spatiale des eaux de rebut dans le Véragne}

17 paramètres physiques et chimiques : $\mathrm{pH}$, Cond. (Conductivité), $\mathrm{T}$ (température), $\mathrm{O}_{2}$ (oxygène dissous), $\mathrm{SiO}_{2}$ (Silice), Cl- (chlorures), Dt. (Dureté

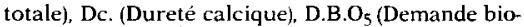
chimique en oxygène en 5 jours), Al. (Alcalinité), $\mathrm{NO}_{2}^{-}$(Nitrites), $\mathrm{NO}_{3}^{-}$(Nitrates), N.O.P. (Azote organique particulaire), la forme assimilable du phosphore : $\mathrm{PO}_{4}{ }^{3-}$ (Orthophosphates), M.E.S. (Matières en suspension, O.P.C. (Oxydabilité au permanganate à chaud) et C.O.P. (Carbone organique particulaire) permettent de caractériser le rejet station Rv (fig. 1). Les effluents sont évacués dans le Véragne sans épuration préalable après avoir reçu les eaux canalisées qui alimentent un moulin $(\mathrm{Cm})$.

Ce canal est une dérivation du Buëch au niveau d'Eyguians. Trois stations situées sur le Véragne $\left(V_{2}, A_{14}\right.$ et $\left.V_{3}\right)$ en aval de la prise en charge des effluents domestiques et des eaux du canal du moulin permettent d'appréhender l'évolution spatiale de la charge polluante sur une portion de cours réduite $(80 \mathrm{~m})$ et de connaître l'importance de cette charge arrivant au Buëch.

\subsection{Evolution temporelle des eaux de rebut}

Quatre formes de l'azote: $\mathrm{NH}_{4}+$ (ion ammonium), $\mathrm{NO}_{2}^{-}, \mathrm{NO}_{3}^{-}$, N.O.P., et $\mathrm{PO}_{4}{ }^{3-}$, O.P.C., C.O.P. et $\mathrm{Cl}^{-}$, paramètres plus spécialement caractéristiques des rejets d'eaux usées domestiques ont été analysés au cours d'une journée, entre 6 h et $22 \mathrm{~h}$ avec une périodicité d'une heure, sur deux stations, l'une prise sur le Véragne en $V_{2}$, l'autre en aval de la confluence du Véragne et du Buëch en B12. Le temps de transit de la masse d'eau entre les deux points étant de l'ordre de $30 \mathrm{mn}$., les prélèvements effectués en B12 ont été décalés d'autant.

\subsection{Evolution spatio-temporelle de la charge pol- luante dans le cours axial}

Six stations ont été choisies de façon à encadrer la source de pollution (fig. 2). Ce sont les stations

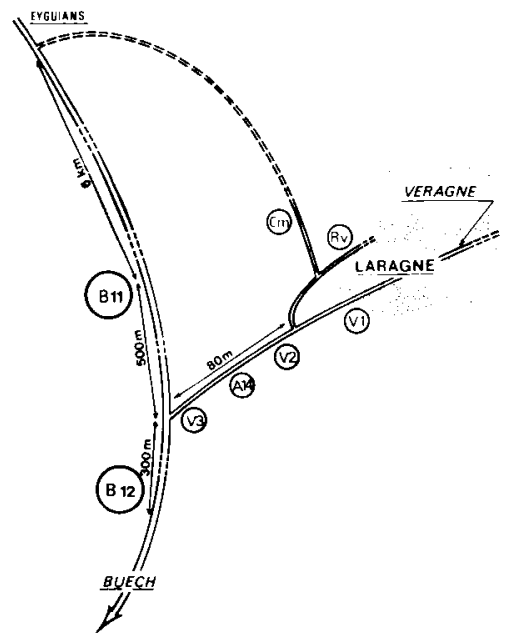

Fig. 1. Schéma du réseau des eaux de rebut de Laragne $(\mathrm{Cm}$ $=$ canal du moulin, $R_{v} .=$ rejets de la ville, $V_{1} \ldots V_{3}=$ stations prises sur le Vèragne, B11 et B12 = stations prises sur le Buëch).

$A_{14}$ au niveau du collecteur des eaux résiduaires de la ville de Laragne (Le Véragne), $B_{11}$ et $B_{12}$ respectivement situées en amont et en aval immédiat de la confluence du Véragne et du Buëch ; les stations $\mathrm{B}_{13}$ et $\mathrm{B}_{14}$ situées à 4 et 10 kilomètres plus en aval. Ces dernières permettent d'évaluer la longueur de rivière touchée par cette pollution organique et d'analyser l'intensité des phénomènes d'autoépuration liés au cycle hydrologique de la rivière. De plus. la station $B_{6}$ localisée sur le Buëch à $20 \mathrm{~km}$ en amont du déversement des eaux usées nous sert de référence (dans les A.C.P. de notre étude précédente portant sur l'ensemble des stations du cours d'eau, le a point-station " B6 était très voisin de l'origine des axes dans le plan factoriel I-II mettant ainsi en évidence, d'une part, l'absence de particularités liées à la minéralisation naturelle des eaux de la station et d'autre part, le faible volume des apports exogènes eutrophisants). 


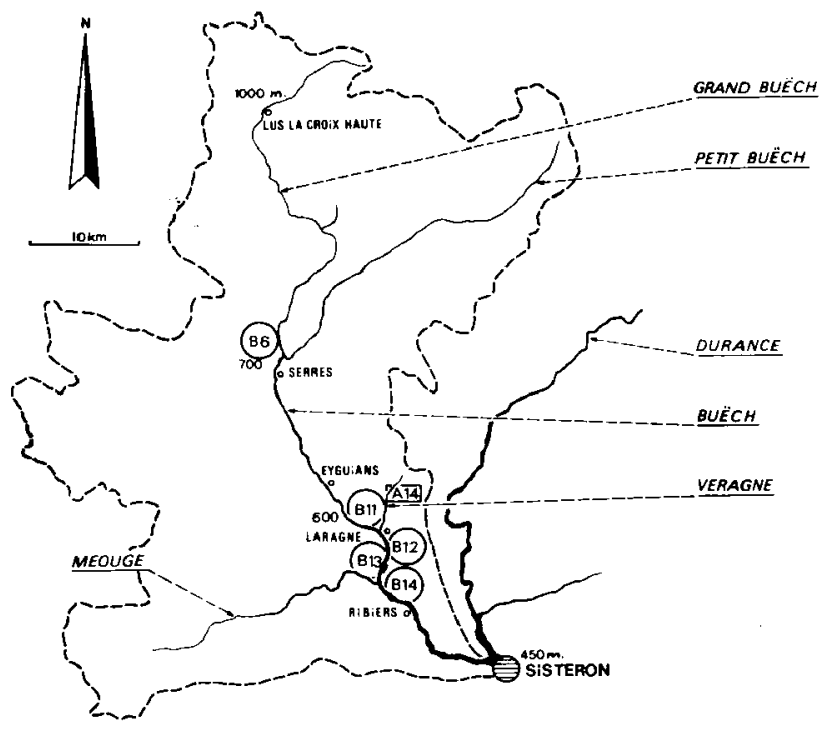

Fig. 2. Le bassin versant du Buẹch : localisation des stations d'étude.

Ces six stations sont suivies pendant la durée d'un cycle hydrologique. Les descripteurs retenus pour caractériser la masse d'eau sont :

$-\mathrm{NO}_{2}^{-}, \mathrm{NO}_{3}^{-}$, N.O.P., $\mathrm{PO}_{4}^{3-}$, O.P.C., C.O.P., D.B. $\mathrm{O}_{5}, \mathrm{Cl}^{-}$

Les descripteurs retenus pour caractériser les sédiments sont :

- le carbone organique (C.O.) et l'azote (N.).

En outre, le $\mathrm{pH}$, la Cond., l' $\mathrm{O}_{2}$, les Dt. et Dc., l'Al., M.E.S. et $\mathrm{SiO}_{2}$ sont précisés dans les six stations.

\section{Caractéristiques physiques et chimi- ques des eaux de rebut}

Le système d'égouttage de la ville de Laragne est simple et non séparatif. Un seul collecteur reçoit à la fois les eaux usées et les eaux de pluie de l'ensemble de l'agglomération qui possède une population permanente de 3900 habitants et un abattoir de moutons. Le débit des rejets varie donc d'une part, en fonction de la saison (pluies ou déficit hydrique) et d'autre part, en fonction de la périodicité journalière des activités domestiques.

\subsection{Evolution spatiale dans le Véragne}

Un prélèvement effectué au niveau du rejet de la ville (Rv) révèle que les éléments qui traduisent plus spécialement une pollution domestique tels $\mathrm{PO}_{4}{ }^{3-}$, O.P.C., N.O.P., D.B.O . $_{5}$ présentent des valeurs très élevées mais aussi, que la concentration des autres composés est importante (Tableau I).

Les concentrations baissent sensiblement entre les stations $\mathrm{Rv}$ et $\mathrm{A}_{14}$. On note une légère hausse en $V_{3}$ due à un phénomène de "reflux *. 
Tableau I. Caractéristiques physiques et chimiques des eaux parvenant au Véragne et des eaux du Véragne lui-mème en aval de Laragne (janvier 1983).

\begin{tabular}{|c|c|c|c|c|c|c|c|c|c|c|c|c|c|c|c|c|c|}
\hline STATIONS & $\mathrm{pH}$ & Cond. & $T$ & $0_{2}$ & SiO2 & $\mathrm{Cl}-$ & Dt & $D C$ & $\mathrm{DBO}$ & Al & $\mathrm{NO}_{2}^{-}$ & $\mathrm{NO}_{3}^{-}$ & $\mathrm{PO}_{4}^{3-}$ & MES & OPC & $C O P$ & NOP \\
\hline Rv & 7.0 & 606 & 10.4 & 7,5 & 9,15 & 47,0 & 290 & 260 & - & 290 & 0,32 & 4,55 & 8,15 & 237.0 & 27.0 & 24,17 & 2,42 \\
\hline $\mathrm{Cm}$ & 7,8 & 232 & 3,3 & 13,6 & 3,85 & 15,5 & 200 & 180 & 2,42 & 180 & tr & 2,88 & 0,01 & 106.0 & 0,7 & 2,73 & 0,11 \\
\hline V1 & 7,8 & 506 & 0,2 & 13,7 & 5,45 & 14,5 & 510 & 465 & 4,84 & 210 & $t r$ & 5,95 & 0,01 & 61.6 & 0,8 & 5.56 & 0,35 \\
\hline v2 & 7,7 & 38.5 & 1.9 & 13,4 & 5,20 & 23,5 & 390 & 365 & 4,84 & 210 & 0,06 & 4,20 & 0,40 & 26.6 & 3,3 & 21.52 & 0,23 \\
\hline Al4 & 8,0 & 28.6 & 2,9 & 12.9 & 4,35 & 16,5 & 260 & 210 & 4.64 & 200 & tr $r$ & 2,65 & 0.26 & 43.0 & 2.0 & 10,24 & 0,83 \\
\hline v3 & 7,9 & 289 & 2,9 & 13,5 & 4,70 & 16,0 & 260 & 240 & 4,84 & 190 & tr & 2,60 & 0,34 & 47.0 & 3,5 & 3,53 & 0,79 \\
\hline
\end{tabular}

Lorsqu'on se réfère à la concentration des effluents, la charge polluante arrivant dans le Buëch au niveau de la station $B_{12}$ est, du fait des dilutions successives, nettement plus réduite. Au moment de la collecte des échantillons, le débit du Véragne en $A_{14}$ était de $1,3 \mathrm{~m}^{3 / 3}$, celui du Buëch (au niveau de la station $B_{11}$ était de $9,1 \mathrm{~m}^{3 /} / \mathrm{s}$; la dilution apportée $p a r$ les eaux du Buëch est donc très importante (fig. 3).

\subsection{Evolution des principaux paramètres hydrochi- miques entre les rejets (Véragne) et le Buëch}

Comme le montre la figure 3 , la charge fluctue énormément au cours d'un cycle journalier; ces fluctuations sont rapidement amorties vers l'aval essentiellement par suite de phénomènes de dilution et de dispersion mais aussi par suite de phénomènes de sédimentation et de bioépuration.

\subsubsection{L'AZOTE}

Comme dans la plupart des effluents urbains, l'azote se trouve essentiellement sous sa forme ammoniacale : urée, acide urique, protéines...

- Les valeurs de $\mathrm{NH}_{4}+($ fig. 3.A), sont faibles à $6 \mathrm{~h}$ du matin, elles s'élèvent progressivement pour atteindre un maximum a $10 \mathrm{~h}$. La concentration baisse ensuite lentement dans le cours de l'aprèsmidi et dans la soirée. Deux pics de moindre importance sont notables à $16 \mathrm{~h}$ et à $20 \mathrm{~h}$. Les valeurs rele. vées à $22 \mathrm{~h}$ sont encore légèrement supérieures à celles enregistrées à $6 \mathrm{~h}$. Dans les effluents qu'il étudie, Vanderborght (1980) constate que les concentrations se stabilisent entre $24 \mathrm{~h}$ et $6 \mathrm{~h}$. Comme dans la Semois, les variations journalières de $\mathrm{NH}_{4}{ }^{+}$dans le Véragne sont en relation avec le rythme de l'activité humaine.
- Les teneurs en N.O.P. (fig. 3.B) suivent sensiblement les mêmes fluctuations. Cependant, le pic de $20 \mathrm{~h}$ est beaucoup plus marqué que celui relevé dans l'évolution de la teneur en $\mathrm{NH}_{4}{ }^{+}$. Les concentrations dosées sont environ 12 fois plus faibles que celles mesurées pour le $\mathrm{NH}_{4}{ }^{+}$. Ceci peut être la conséquence d'un phénomène d'ammonification qui a lieu en grande partie dans le réseau d'égout : Vanderborght (1980) signale en effet qu'en fin de réseau, les proportions peuvent atteindre jusqu'à $80 \%$ de $\mathrm{NH}_{4}{ }^{+}$et $20 \%$ de N.O.P. Ensuite cette ammonification se poursuit dans le Véragne.

- Les concentrations en $\mathrm{NO}_{3}{ }^{-}$(fig. 3.C) relevées au cours du cycle journalier, restent relativement constantes bien qu'une chute brutale de la concentration à $16 \mathrm{~h}$, soit observée sans explication. Le signal complexe enregistré s'explique par un mélange d'eaux :

- eaux de ruissellement agricole;

- eaux de pluie dans le système d'égout ;

- eaux domestiques, avec passage de la forme $\mathrm{NH}_{4}{ }^{+}$à la forme $\mathrm{NO}_{3}{ }^{-}$par nitrification (aussi bien dans le réseau d'égouttage que dans la rivière).

Vanderborght a effectué des mesures directement dans le système d'égout et conclut que $\mathrm{NO}_{3}{ }^{-}$présente une courbe d'allure inverse de celle de $\mathrm{NH}_{4}{ }^{+} . \mathrm{NO}_{3}{ }^{-}$prédomine: les concentrations dosées sont en effet environ 3 fois supérieures à cel. les enregistrées pour $\mathrm{NH}_{4}{ }^{+}$. Dans le Véragne, les fortes concentrations en $\mathrm{NO}_{3}{ }^{-}$mesurés au niveau de la station $\mathrm{V}_{2}$, proviennent en partie de l'oxydation de $\mathrm{NH}_{4}{ }^{+}$et $\mathrm{NO}_{2}{ }^{-}$; mais si l'on se réfère au Tableau I, on constate que $\mathrm{NO}_{3}{ }^{-}$seulement est véhiculée en grande quantité par le ruisseau luimême en $V_{1}$. 
(5)

ECOLOGIE DUNE RIVIERE NON AMÉNAGÉE DES ALPES DU SUD

7
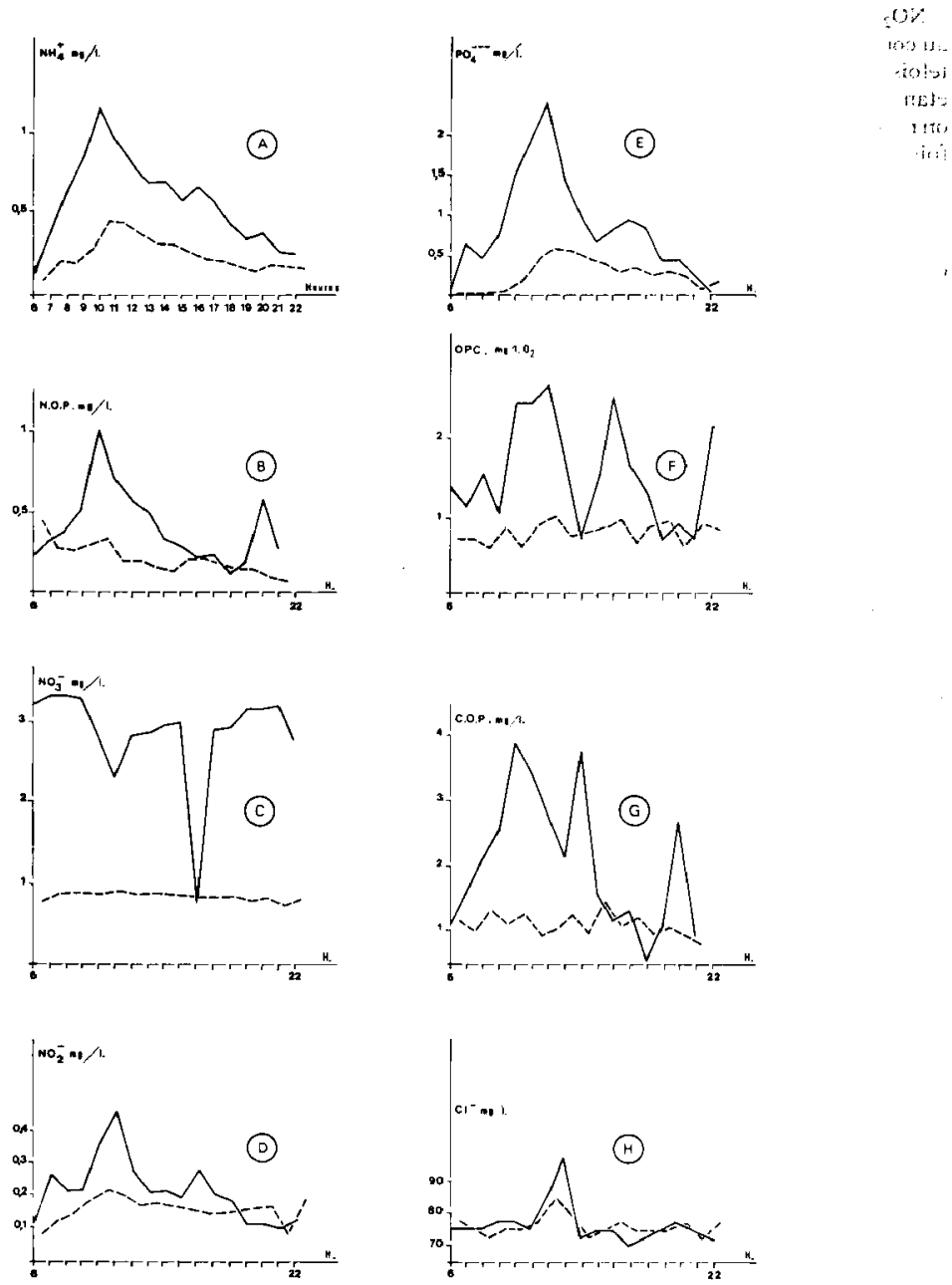

Fig. 3. Evolution journalière comparée des descripteurs des eaux du Véragne en Al4 (courbes en trait plein) et du Buëch au niveau de la station B12 (courbes en trait interrompu). 
$\mathrm{NO}_{2}{ }^{-}$(fig. 3.D) présente le même type d'évolution au cours de la journée que $\mathrm{NH}_{4}+$. On constate toutefois, que le maximum a lieu à $11 \mathrm{~h}$, la décroissance étant ensuite brutale dès le début de l'après-midi ; on note un nouveau pic à $16 \mathrm{~h}$, son amplitude est 2,5 fois moindre que celle observée pour $\mathrm{NH}_{4}{ }^{+}$.

\subsubsection{LES ORTHOPHOSPHATES}

L'évolution de la concentration en $\mathrm{PO}_{4}{ }^{3-}$ (fig. 3.E) est semblable à celle du $\mathrm{NH}_{4}{ }^{+}$. La teneur maximum est relevée à $12 \mathrm{~h}$. Un second pic, moins marqué, apparaît à $17 \mathrm{~h}$. Vanderborght constate que l'amplitude du signal du lundil est régulièrement plus élevée. Elie permet de reconnaître le jour habituel de lessive, car dans les eaux usées ménagères, les composés du phosphore sont associés en grande partie aux détergents.

\subsubsection{L'OXYDABILITE}

L'O.P.C. donne une idée globale de la concentration en matières organiques et en substances réductrices. La charge de l'effluent atteint son maximum en fin de matinée : de 10 à 12 h (fig. 3.F) elle décroit ensuite fortement en début d'après-midi, marque un second pic à $16 \mathrm{~h}$, puis un troisième aux environs de $22 \mathrm{~h}$.

\subsubsection{LE CARBONE ORGANIOUE PARTICULAIRE}

La courbe représentant les variations journalières du C.O.P. (fig. 3.G), possède la même allure que celle tracée pour l'évolution de l'O.P.C., mais les 3 pics sont plus précoces puisqu'ils apparaissent $2 \mathrm{~h}$ avant.

\subsubsection{LES CHLORURES}

Comme pour les concentrations en $\mathrm{PO}_{4}{ }^{3-}$, le maximum se situe en fin de matinée (fig. 3.H). Les $\mathrm{C} 1$ - sont très facilement solubles, ils ne jouent aucun rôle dans les phénomènes de décomposition et ne sont donc pas modifiés (Rodier 1978). Il est donc normal de ne pas constater d'amortissement entre les stations $V_{2}$ et $B_{12}$ sauf au niveau du pic (on incriminera alors un processus de dilution).

Les résultats obtenus à la suite des analyses d'eau réalisées toutes les heures sont en parfaite concordance avec les observations faites par Vanderborght (1980) sur la Sémois durant une semaine.

1. Les prélèvements ont été réalisés, dans le Véragne, le lundi 22 aoùt 1983.
L'amortissement vers l'aval des fluctuations des différents descripteurs met l'accent sur l'importance des phénomènes de dilution et de sédimentation.

\section{Evolution spatio-temporelle de la charge polluante dans le Buëch}

L'évolution des conditions abiotiques du Buèch, en amont et en aval de Laragne, est analysée au moyen d'une A.C.P. (analyse en composantes principales). Par suite des fluctuations journalières constatées au niveau des descripteurs choisis, tous lés tichantillons d'eau ont été prélevés sensiblement à la même heure. Aux cinq stations prospectées sur le cours principal $\left(B_{6}, B_{11}, B_{12}, B_{13}\right.$ et $\left.B_{14}\right)$. est ajoutée une station du Véragne $\left(A_{14}\right)$ : elle permet de définir la charge polluante arrivant dans le Buëch (Tableau II).

La part de variance cumulée esı de $60,48 \%$ sur les deux premiers axes principaux : $37,23 \%$ sur le premier, $23,25 \%$ sur le second. Parmi les 17 descripteurs retenus pour l'étude, le $\mathrm{pH}$ possède une contribution significative seulement sur l'axe IV, ce descripteur ne sera donc pas pris en considération dans l'interprétation des résultats.

\subsection{Interprétation du premier axe principal}

La matrice de corrélation entre les variables et les composantes principales montre que seul $\mathrm{O}_{2}$ présente une corrélation négative avec la composante principale $C_{1}$. A l'opposé, les plus fortes valeurs de corrélation positive relevées avec le premier axe sont, pour les descripteurs du milieu aqueux : le $\mathrm{PO}_{4}{ }^{3-}$, l'O.P.C., la $\mathrm{DBO}_{5}$ et le N.O.P. et pour les sédiments, le C.O. Le premier axe principal est donc entièrement déterminé, d'une part, par la matière organique au sens large, (qui s'oppose à la concentration en $\mathrm{O}_{2}$ ) et d'autre part, par les $\mathrm{PO}_{4}{ }^{3-}$. Il décrit donc l'intensité de la pollution domestique (à la fois dans l'eau et dans les sédiments).

Certaines variables telles : O.P.C., C.O.P., $\mathrm{PO}_{4}{ }^{3-}$, N.O.P. sont fortement corrélées entre elles (Tableau III).

La D.B.O. 5 fortement corrélée avec la composante $C_{1}$, est à la fois corrélée aux descripteurs du milieu aqueux et au C.O. du sédiment (les coefficients sont 
Tableau II. Résultats des analyses effectuées au cours d'un cycle hydrologique dans les 5 stations du Buëch et dans le Véragne.

\begin{tabular}{|c|c|c|c|c|c|c|c|c|c|c|c|c|c|c|c|c|c|c|}
\hline \multicolumn{8}{|c|}{$J A N \cup I E R$} & \multicolumn{5}{|c|}{ HANS } & \multicolumn{6}{|c|}{$M A$} \\
\hline & - 86 & B11 & $\$ 12$ & 3 & $B 14$ & A14 & B6 & B11 & 812 & B13 & 314 & 16 & - $B 6$ & 11 & 12 & 113 & 14 & \\
\hline & 180 & 160 & 190 & 18 & 185 & 240 & 190 & 175 & 190 & 170 & 160 & 35 & 190 & 180 & 185 & 180 & 180 & 220 \\
\hline Dt. & 185 & 200 & 200 & 200 & 195 & 260 & 230 & 215 & 215 & 220 & 210 & 290 & 200 & 200 & 200 & 200 & 190 & 250 \\
\hline cond. & 319 & 364 & 381 & 383 & 378 & 769 & 432 & 436 & 437 & 466 & 468 & 654 & 315 & 332 & 340 & 335 & 337 & 429 \\
\hline pH & 7.6 & 7,6 & 7,7 & 7 & 7,6 & 3. & 8,0 & 8,1 & 8,0 & 7.9 & 7,9 & 8 & .9 & 8,0 &, 9 & 8,0 & 0,0 & 3,3 \\
\hline T. & 3,0 & 0,6 & 2,9 & 2.0 & 1,5 & 2,9 & 13,2 & 13,5 & 13.8 & 9.6 & 8.9 & 7,3 & 10.1 & 13.0 & 11,9 & 13.4 & 13.9 & 13.7 \\
\hline $\mathrm{O}_{2}$ & 13,9 & 14,0 & 13,8 & 14,1 & 13,6 & 13,5 & 10,6 & 10,5 & 10,0 & 11,5 & 11,6 & 11,5 & 10,3 & 13,8 & 10,9 & 11,6 & 11.6 & 1,1 \\
\hline sat & 100 & 100 & 100 & 100 & 100 & 100 & 100 & 100 & 100 & 100 & 100 & 98 & 100 & 100 & 100 & 100 & 100 & 100 \\
\hline Al. & 160 & 180 & 180 & 180 & 180 & 190 & 190 & 190 & 195 & 190 & 190. & 220 & 185 & 185 & 185 & 180 & 180 & 195 \\
\hline WES & 12,6 & 23,4 & 17,2 & 8,8 & 7,8 & 47,0 & 11,6 & 57,4 & 41,0 & 36,0 & 61,4 & 85,6 & 7,0 & 23,6 & 4,6 & 22,4 & 30,6 & 8.2 \\
\hline OPC & 0,65 & 0,75 & 0,50 & 0,80 & 0.55 & 3,50 & 0,52 & 0,57 & 1,22 & 0,62 & $0,70$. & 7,30 & 0.52 & 0.42 & 0.37 & 0.45 & 0,45 & 0,80 \\
\hline $1-$ & 7.0 & 17.5 & 18.5 & 18.5 & 16.5 & 16,0 & 7,0 & 9.0 & 9.0 & 9.0 & 8.0 & 10,0 & 7,0 & 9,5 & 9,5 & 9,5 & 8,5 & .0 \\
\hline $\mathrm{NO}_{2}$ & cr. & er. & 0,01 & $\tau \tau$ & $t r$. & $t r$ & $t r$ & tx & te & tr & $t 5$ & tr. & tr. & $t r$ & $t \tau$ & tr. & tr. & $t r$. \\
\hline Noj & 1,12 & 2,44 & 2,12 & 2,38 & 2,16 & 2,60 & 1,82 & 1,45 & 1,45 & 1,62 & 1,52 & 2,61 & 1,49 & 1,59 & 1.63 & 1,66 & 1,85 & 2,16 \\
\hline $\mathrm{O}_{4}^{3-}$ & 0,01 & 0,01 & 0,05 & 0,04 & 0,02 & 0.34 & 0.01 & 0,01 & 0,0 & 0,01 & 0.01 & 1,02 & 0.01 & 0,01 & 0.01 & 0.01 & 0,01 & 0,27 \\
\hline BOS & 2,01 & 2,22 & 2,22 & 2,22 & 2,42 & 4,84 & 2,62 & 2,82 & 2,72 & 3,02 & 3,12 . & 5,84 & - & - & - & - & - & - \\
\hline COP & 0.47 & 0.26 & 0,37 & 0,30 & 0,29 & 3.53 & 0,92 & 1,16 & 1,72 & 1,00 & 1,04 & 10,47 & 0,30 & 0.10 & 0,20 & 0.12 & 0,26 & 1,10 \\
\hline NOP & 0,02 & 0,19 & 0,03 & 0,06 & 0,02 & 0,79 & 0,10 & 0,23 & 0,14 & 0,13 & 0,17 & 0,86 & 0,03 & 0.01 & 0,02 & 0.01 & 0,03 & 0,11 \\
\hline
\end{tabular}

SEDIRENT

co. $\quad 3,77 \quad 3,092,54 \quad 2,67 \quad 6,19 \quad 7,6 \quad 3,40 \quad 3,002,20 \quad 1,94 \quad 3,75 \quad 7,51=$

n. $\quad 0,140,620,33 \quad 0,360,56 \quad 1,46 \quad 0,10 \quad 0,090,16 \quad 0,55 \quad 1,67 \quad 1,44$ -

EAU . B6 B11 B12 BIS B14 A14. B6 B11 812 B13 B14. A14. B6

Dc. $\quad \begin{array}{lllllllllllll}150 & 165 & 165 & 155 & 150 & 175 & 185 & 175 & 170 & 195 & 155 & 180 & 190\end{array}$

SEPTEMBEE XOVEHBRE

De. $\quad \begin{array}{llllllllllllllllllllllll}180 & 190 & 195 & 195 & 190 & 210 & 210 & 210 & 195 & 215 & 165 & 195 & 225 & 235 & 225 & 225 & 255 & 230 & 205 & 215 & 240 & 225 & 220\end{array}$

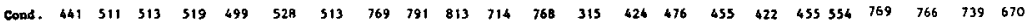

$\begin{array}{lllllllllllllllllllllllllll}\text { pH } & 7,9 & 7,9 & 7,9 & 8,0 & 8,0 & 8,0 & 7,8 & 7,9 & 7,8 & 7,9 & 7,9 & 7,7 & 8,0 & 7,9 & 8,0 & 8,1 & 8,0 & 7,9 & 7,7 & 7,8 & 7,6 & 7,9 & 7,7\end{array}$

I. $\quad 14,4 \quad 18,3 \quad 19,6 \quad 21,1 \quad 21,6 \quad 18,8 \quad 19,2 \quad 20,8 \quad 19,9 \quad 19,5 \quad 20,3 \quad 20,2 \quad 12,8 \quad 21,3 \quad 19,0 \quad 19,3 \quad 19,2 \quad 19,3 \quad 6,1 \quad 6,3 \quad 6,5 \quad 7,5 \quad 8,1$

$\begin{array}{lllllllllllllllllllllllllllll}0 & 11,0 & 10,0 & 10,3 & 10,3 & 9,9 & 10,6 & 9,4 & 8,7 & 7,4 & 9,5 & 0,9 & 6,8 & 11,3 & 9,6 & 10,5 & 11,9 & 9,0 & 9,4 & 14,7 & 12,6 & 12,3 & 12,8 & 13,1\end{array}$

$\begin{array}{llllllllllllllllllllllllllll}\text { I.at. } 100 & 100 & 100 & 100 & 100 & 100 & 100 & 83 & 100 & 100 & 76 & 100 & 100 & 100 & 100 & 100 & 100 & 100 & 100 & 100 & 100 & 100 & 100\end{array}$

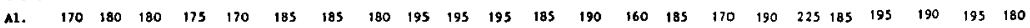

YES $\begin{array}{lllllllllllllllllllllllllllll}8,2 & 5,2 & 16,0 & 17,0 & 2,4 & 15,4 & 2,4 & 45,2 & 17,0 & 15,2 & 3,0 & 12,4 & 1,8 & 8,8 & 12,8 & 8,2 & 5,0 & 5,6 & 0,5 & 1,3 & 1,7 & 1,5 & 2,5\end{array}$

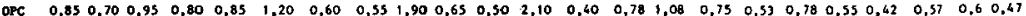

C1- $\quad 7,024,524,0 \quad 24,0 \quad 20,5 \quad 24,5 \quad 10,0 \quad 85,0 \quad 80,0 \quad 52,5 \quad 35,0.77,5 \quad 22,5 \quad 65,0 \quad 86,0 \quad 80,0 \quad 57,5 \quad 97,5 \quad 27,5 \quad 57,5 \quad 55,0 \quad 60,0 \quad 45$

$\begin{array}{lllllllllllllllllllllllllll}0_{7}^{-} & 1,25 & 2,15 & 2,22 & 2,32 & 2,04 & 2,12 & 2,95 & 3,65 & 3,46 & 4,25 & 3,00 & 3,54 & 2,32 & 2,16 & 2,12 & 2,32 & 2,36 & 2,04 & 1,07 & 1,53 & 1,55 & 1,69 & 1,31 & 1,59\end{array}$

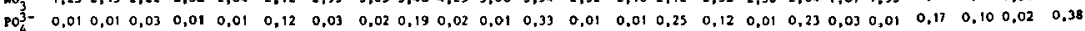

$\begin{array}{lllllllllllllllllllllllllllllll}\text { DBOS } & 0,90 & 1,31 & 1,61 & 2,01 & 0,60 & 5,24 & 3,02 & 3,73 & 3,83 & 2,21 & 2,62 & 6,04 & 1,11 & 1,21 & 3,43 & 2,32 & 1,41 & 6,15 & 1,12 & 3,33 & 5,14 & 4,44 & 3,53 & 0,07\end{array}$

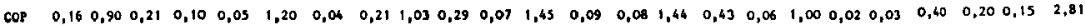

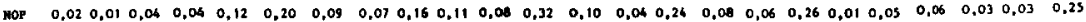

\section{SEDIMENT}

$\begin{array}{llllllllllllllllllllllllll}\text { Co. } \quad 3,36 & 1,75 & 7,56 & 8,19 & 2,56 & 9,44 & 7,16 & 3,95 & 10,767,97 & 7,59 & 22,64 & 4,86 & 8,14 & 8,21 & 6,78 & 5,71 & 5,60 & 0,44 & 4,19 & 6,87 & 6,48 & 5,18 & 11,75\end{array}$

k. $\quad 0,261,71 \quad 2,07 \quad 1,41 \quad 0,75 \quad 1,66 \quad 4,8 \quad 1,002,55 \quad 2,70 \quad 0,86 \quad 3,27 \quad 0,54 \quad 1,55 \quad 1,48 \quad 1,27 \quad 1,10 \quad 0,89 \quad 1,46 \quad 0,39 \quad 0,64 \quad 1,15 \quad 0,64 \quad 2,62$ 
de l'ordre de 0,5). La plupart des autres descripteurs sont bien corrélés avec $\mathrm{C}_{1}$ et participent, mais à un degré moindre, à la détermination de l'axe. Comme nous l'avons vu précédemment lors de l'étude de la composition du rejet, leur concentration est plus importante dans l'effluent que dans la rivière.

Les stations représentées dans le plan principal I/II se répartissent sur l'axe I, de la façon suivante : la station $\mathrm{A}_{14}$ (Véragne) occupe la partie positive de l'axe durant tout le cycle ; les autres stations, toutes situées sur le Buëch, se répartissent d'une part, en fonction de l'époque du prélèvement, d'autre part en fonction de leur position géographique par rapport au Véragne (fig. 4). Ainsi, les points qui représentent les analyses effectuées en janvier, se situent sur la partie négative de l'axe I, en opposition avec les échantillons prélevés en août, plus proches de ceux effectués dans le Véragne. Les prélèvements réalisés au cours des mois de mars, septembre et novembre occupent une position intermédiaire. Les résultats des analyses effectuées en juin se rapprochent de ceux du mois de janvier mais les écarts des coordonnées entre les stations y sont plus grands.
L'axe I met donc en évidence l'importance de l'évolution saisonnière dans un tel hydrosystème. Il traduit une pollution (eutrophication) par des matières organiques, mais aussi une eutrophisation de l'ensemble des stations, notamment au cours de la période estivale.

La station $\mathrm{B}_{13}$ subit de façon moins évidente les effets de l'apport en éléments nutritifs. Son évolution temporelle présente à peu près le même profil que celui de la station $B_{12}$ avec, toutefois, des valeurs plus basses en août et en septembre. La courbe dressée à partir des prélèvements effectués dans la station $\mathrm{B}_{14}$ se rapproche de celle de la station de référence $B_{6}$ : les effets du Véragne n'apparaissent plus à son niveau. La station $B_{14}$ est cependant caractérisée par une plus grande constance dans son niveau d'eutrophie : l'amplitude des variations saisonnières y est plus restreinte. $O$ n note au niveau de la station $B_{11}$, une légère augmentation du niveau d'eutrophie par rapport à la station $\mathrm{B}_{6}$ mais l'allure de la courbe reste la même.

Sauf peut-être en août, $B_{6}$ apparaît bien comme une station de référence. Mais il importe de signaler
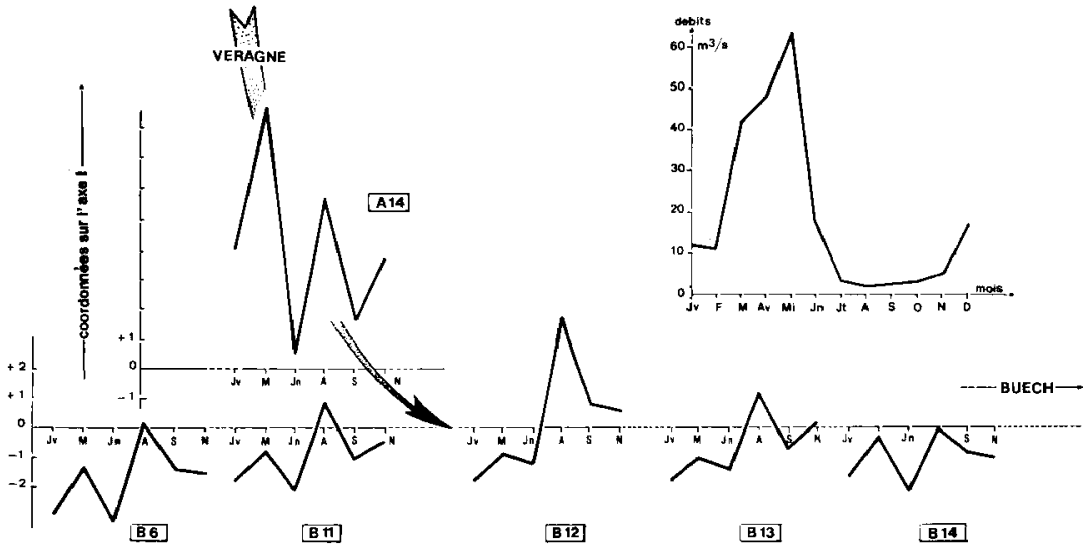

Fig. 4. Impact des eaux du Véragne sur le chimisme du Buëch. L'évolution est suivie à partir des coordonnées des points " station-temps * sur l'axe I (en haut et à droite : courbe temporelle du débit). 


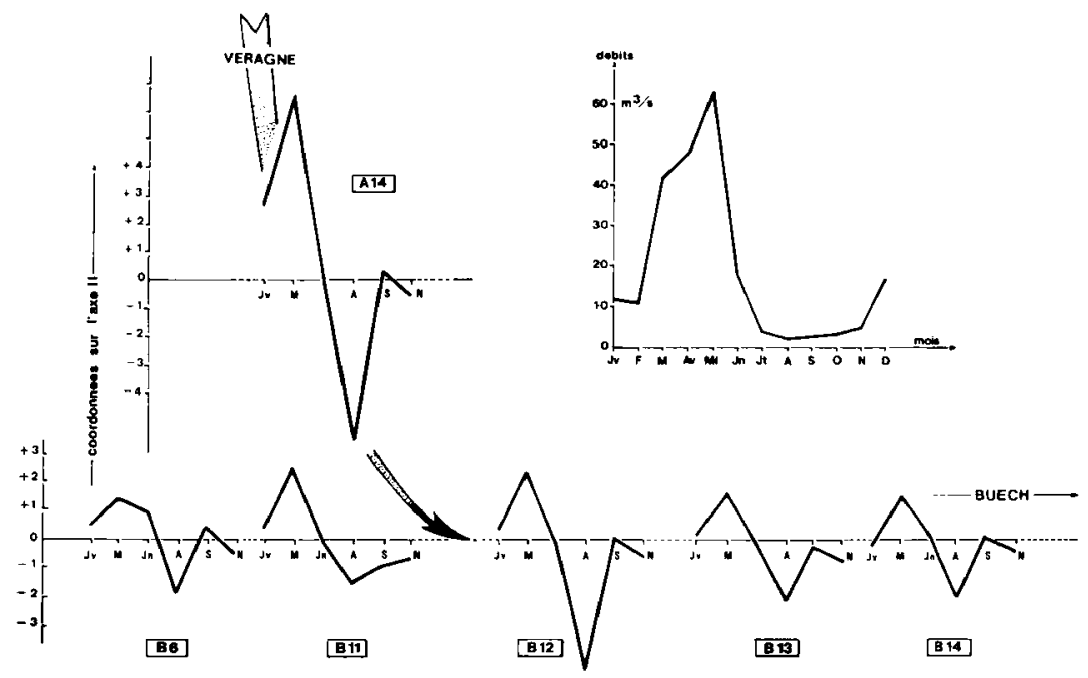

Fig. 5. Impact des eaux du Véragne sur le chimisme du Buëch. L'évolution est suivie à partir des coordonnées des points * station-temps " sur l'axe II (en haut et à droite : courbe temporelle du débit).

que dans un tel hydrosystème, l'évolution saison. nière prime sur l'évolution spatiale : la station $\mathbf{B}_{6}$ par exemple, présente en août un degré d'eutrophie supérieur à celui observé dans la station $\mathbf{B}_{12}$ au cours des mois de janvier, mars et juin.

\subsection{Interprétation du second axe principal}

Sur l'axe Il s'opposent d'une part, les descripteurs dont la teneur augmente par dépôt ou par concentration lorsque le débit est faible $\left(\mathrm{NO}_{\overline{2}}, \mathrm{NO}_{3}, \mathrm{Cl}-\right)$ et d'autre part, ceux dont les valeurs augmentent avec le débit, soit par lessivage (Dt.) soit par remise en suspension (C.O.P., N.O.P., $\mathrm{PO}_{4}{ }^{3-}$, O.P.C.) 1 soit, enfin, par suite du brassage plus important de l'eau $\left(\mathrm{O}_{2}\right)$.

1. Les teneurs en orthophosphates augmentent en début de crue, $\mathrm{PO}_{4}{ }^{3-}$ - fait partie des anions facilement fixés par le sol.
Il est évident qu'au delà d'une certaine limite, le débit continuant d'augmenter, les concentrations des différents éléments dans l'eau chuteraient : c'est ce que confirment les résultats des mesures effec. tuées au cours du mois de mai (pleine crue). Ces résultats n'ont pas été pris en compte dans l'analyse, certains descripteurs n'ayant pas pu être échantillonnés.

Les mois de mars et d'août s'opposent sur l'axe II ( $f$ ig. 5) et mettent ainsi en relief l'importance des phénomènes liés à l'hydraulique.

En août, au cours de l'étiage estival, un phénomène de sédimentation prédomine au niveau de toutes les stations; il est particulièrement sensible au niveau des stations $A_{14}$ et $B_{12}$ dont les positions sont très négatives, une partie importante de la charge véhiculée par les eaux du Vèragne se dépose avant la confluence, une autre partie parvient au Buëch et se dépose au niveau de la station $B_{12}$. 
Tableau III. Matrice de corrélation entre les variables (Légendes : $\mathrm{Dt}=$ Dureté totale, Al. $=$ Alcalinité, MES $=$ Matières en suspension, $\mathrm{OPC}=$ oxydabilité au permanganate à chaud, Cond. = Conductivité, $\mathrm{COP}=\mathrm{Carbone}$ organique particulaire, NOP = Azote organique particulaire, CO. Sed = Carbone organique du sédiment, N. Sed = Azote organique du sédiment).

\begin{tabular}{|c|c|c|c|c|c|c|c|c|c|c|c|c|c|c|c|c|c|}
\hline Dt & $\begin{array}{c}\text { Dt } \\
1.000\end{array}$ & $\mathrm{DH}$ & $0_{2}$ & Al & $\mathrm{SiO}_{2}$ & MES & OPC & $\mathrm{Cl}-$ & $\mathrm{MO}_{2}^{-}$ & OBO & Cond & $\mathrm{NO}_{3}^{-}$ & $\mathrm{PO}_{4}^{3-}$ & COP & NOP & Co.5ed & N. Seo. \\
\hline $\mathrm{pH}$ & 0.162 & 1.000 & & & & & & & & & & & & & & & \\
\hline $0_{2}$ & 0.189 & 0.173 & 1.000 & & & & & & & & & & & & & & \\
\hline A1 & 0.487 & 0.126 & -0.084 & 1.000 & & & & & & & & & & & & & \\
\hline $\mathrm{SiO}_{2}$ & 0.170 & -0.183 & -0.340 & 0.312 & 1.000 & & & & & & & & & & & & \\
\hline MES & 0.370 & 0.264 & -0.000 & 0.320 & -0.010 & 1.000 & & & & & & & & & & & \\
\hline$O P C$ & 0.518 & 0.133 & -0.250 & 0.430 & 0.106 & 0.637 & 1.000 & & & & & & & & & & \\
\hline $\mathrm{Cl}-$ & 0.109 & 0.036 & -0.356 & 0.172 & 0.536 & -0.313 & -0.111 & 1.000 & & & & & & & & & \\
\hline $\mathrm{NO}_{2}^{-}$ & -0.192 & -0.219 & -0.931 & 0.130 & 0.391 & -0.071 & 0.154 & 0.424 & 1.000 & & & & & & & & \\
\hline DBO & 0.511 & -0.202 & -0.309 & 0.626 & 0.332 & 0.265 & 0.423 & 0.353 & 0.381 & 1.000 & & & & & & & \\
\hline Cond & 0.200 & -0.092 & -0.363 & 0.370 & 0.531 & 0.056 & 0.271 & 0.487 & 0.496 & 0.573 & 1.000 & & & & & & \\
\hline $\mathrm{NO}_{\overline{3}}$ & -0.037 & 0.076 & -0.444 & 0.140 & 0.467 & 0.073 & 0.219 & 0.392 & 0.514 & 0.111 & 0.403 & 1.000 & & & & & \\
\hline $\mathrm{PO}_{4}^{3-}$ & 0.634 & 0.022 & -0.276 & 0.556 & 0.225 & 0.493 & 0.915 & 0.123 & 0.219 & 0.662 & 0.364 & 0.175 & 1.000 & & & & \\
\hline COP & 0.639 & 0.126 & -0.110 & 0.536 & 0.091 & 0.706 & 0.952 & -0.150 & 0.037 & 0.521 & 0.219 & 0.082 & 0.930 & 1.000 & & & \\
\hline NOP & 0.613 & 0.211 & $=0.196$ & 0.522 & 0.169 & 0.662 & 0.881 & -0.048 & 0.113 & 0.562 & 0.313 & 0.200 & 0.847 & 0.877 & 1.000 & & \\
\hline cosed & 0.110 & -0.142 & -0.741 & 0.130 & 0.446 & -0.073 & 0.282 & 0.525 & 0.736 & 0.558 & 0.532 & 0.502 & 0.430 & 0.195 & 0.312 & 1.000 & \\
\hline NSed & 0.071 & -0.101 & -0.417 & 0.111 & 0.356 & -0.064 & 0.154 & 0.247 & 0.524 & 0.293 & 0.411 & 0.539 & 0.244 & 0.094 & 0.154 & 0.637 & 1,000 \\
\hline
\end{tabular}

En mars, avec l'augmentation du débit se produit une remise en suspension des éléments qui s'étaient accumulés ou développes sur le fond de la rivière (dépôts organiques, algues, mic roorganismes bactériens...) pour toutes les stations, mais aussi parce que le système des eaux est non séparatif, des dépôts accumulés dans les conduites. L'ensemble provoque une brusque augmentation de la charge polluante. Cette charge parvient au Buëch où elle est immédiatement diluée par les eaux grossies par la fonte des neiges et les pluies printanières; elle n'apparait alors pas plus importante au niveau de la station $B_{12}$ que dans les autres stations à la même époque.

A la fin des hautes eaux, les phénomènes de sédimentation prennent à nouveau de l'importance.

En conséquence, l'axe II, isolé par l'A.C.P., reflète le rôle du débit dans la répartition de la charge organique entre l'eau et le sédiment.

S'il existe effectivement une zone de biodégradation, qui commence dans le réseau d'égout lui-même et se poursuit dans le Véragne, sa longueur est faible : $80 \mathrm{~m}$ environ jusqu'à la confluence du Véragne et du Buëch (fig. 1). La zone de décomposition qui devrait lui faire suite dans le Buëch n'est perçue que pendant l'été (Tableau III). En effet, si pendant l'étiage hivernal et pendant les hautes caux de la rivière, la D.B.O.5 reste importante dans le Véragne, les valeurs mesurées en aval de la confluence BuëchVéragne sont faibles et voisines de celles mesurées en amont de la confluence. Quelles que soient les stations considérées, les eaux sont toujours bien oxygénées. On peut alors penser qu'à cette époque du cycle hydrologique, la régulation du chimisme des eaux est plus mécanique (dilution, sédimentation,...) que biologique (autoépuration).

En revanche, au cours de l'étiage estival, on note au niveau de la station $B 12$, la présence d'un important développement bactérien qui s'accompagne de fortes valeurs prises par la D.B.O.5. La valeur de la D.B.O.5 décroît ensuite progressivement depuis le Véragne jusqu'à la station B14. Corrélativement, on observe une légere diminution de la teneur en $\mathrm{O}_{2}$ dans les eaux de la station $\mathrm{B} 12$ (minimum $83 \%$ en août). Dans les autres stations $\mathrm{O}_{2}$ se maintient à saturation. 
L'oxydation des composés azotés qui se produit plus tard que celle des composés carbonés est décelable: present dans le rejet, l'azote sous forme réduite est transformé en $\mathrm{NO}_{3}-$ entre le niveau des rejets dans le Véragne et la station B13. En aout, par exemple, les concentrations en $\mathrm{NH}_{4}{ }^{+}$diminuent progressivement entre le Véragne et la station $\mathbf{B} 12$; l'augmentation des $\mathrm{NO}_{2}$ - à la station $\mathrm{B} 12$, puis des $\mathrm{NO}_{3}{ }^{-}$à la station $\mathrm{B} 13$, met en évidence un phénomène de nitrification.

\section{Conclusion}

A l'image de la plupart des systèmes lotiques, il apparaît que la composition chimique de l'eau change graduellement de l'amont vers l'aval dans l'hydrosystème Buëch. La minéralisation et le degré d'eutrophie augmentent au fur et à mesure que croit la superficie du bassin versant (Vespini, Légier \& Champeau 1987). A cette eutrophisation perçue à l'échelle du cours d'eau, se surajoute un certain nombre de perturbations locales, qui peuvent être chroniques comme c'est le cas des déversements d'eaux usées ménagères ou provenant d'abattoirs dans le secteur de Laragne. Mais ces phénomènes d'eutrophication, sensibles et même aigus à l'échelle de l'affluent (Véragne), ne semblent pas avoir, dans le Buëch, un impact comparable à celui observé dans d'autres cours d'eau de la région comme l'Eau
Salée (affluent de l'Argens) dans le Var (Dehzad 1978 puis Giudicelli et al. 1980) ou dans le Tavignano en Corse (Giudicelli, Légier, Musso \& Prévot 1986).

Dars le réseau hydrographique du Buëch, la zone critique correspond au secteur inférieur du Véragne, la capacité d'autoépuration de l'affluent est limitée par sa faible longueur $(80 \mathrm{~m})$. Une charge organique encore élevee parvient au Buëch, elle y subit une dilution importante. Le caractère des phénomènes observés est alors essentiellement mécanique et masque le processus de biodégradation sauf pendant les basses eaux estivales.

\section{Travaux cltés}

Dehzad (B.). 1978. - Etude écologique d'un cours d'eau pollué : " l'eau Salée "Var). Thèse de 3ème cycle, Aix Marseille III, $147 \mathrm{p}$.

Giudicelli (J.). Dia (A.). \& Légier (P.). 1980. - Etude hydrobiologique de l'Argens (Var) : peuplements invertébrés et distribution longitudinale. Bijdr. Dierkd., 50:303-341.

Giudicelli (J.), Légier (P.), Musso (J.J.) \& Prévot (G.).1986. - Impact d'une perturbation polluante d'origine organique sur les populations et les communautés d'invertébrés benthiques et en dérive d'une rivière méditerranéenne, le Tavignano (Corse). Ecologia Mediterranea, XII, 1-2: 31-61.

Vanderborght (P.). 1980. - Etude physicochimique des eaux de la Semois. Thèse. Arlon Fond. Univ. Ltuxemb. : 264 p.

Vespini (F.), Legier (P.) \& Champeau (A.). 1987, - Ecologie d'une rivière non aménagée : Le Buech (France), I. Evolution longitudinale des descripteurs physiques et chimiques. Annls. Lim. nol., 23(2): 15t-164. 